Bull. Korean Math. Soc. 50 (2013), No. 3, pp. 811-821

http://dx.doi.org/10.4134/BKMS.2013.50.3.811

\title{
GLOBAL SOLUTIONS OF THE EXPONENTIAL WAVE EQUATION WITH SMALL INITIAL DATA
}

\author{
HyungJin Huh
}

\begin{abstract}
We study the initial value problem of the exponential wave equation in $\mathbb{R}^{n+1}$ for small initial data. We shows, in the case of $n=1$, the global existence of solution by applying the formulation of first order quasilinear hyperbolic system which is weakly linearly degenerate. When $n \geq 2$, a vector field method is applied to show the stability of a trivial solution $\phi=0$.
\end{abstract}

\section{Introduction}

We are interested in the initial value problem of the following exponential wave equation

(1.1)

$\frac{\partial}{\partial t}\left(\partial_{t} \phi \exp \left(\frac{1}{2}|\nabla \phi|^{2}-\frac{1}{2}\left(\partial_{t} \phi\right)^{2}\right)\right)-\sum_{i=1}^{n} \frac{\partial}{\partial x_{i}}\left(\partial_{i} \phi \exp \left(\frac{1}{2}|\nabla \phi|^{2}-\frac{1}{2}\left(\partial_{t} \phi\right)^{2}\right)\right)=0$,

where $\phi: \mathbb{R}^{n+1} \rightarrow \mathbb{R}$ is a scalar function.

An exponentially harmonic map was introduced by J. Eells and L. Lemaire $[3,4]$, which generalizes usual harmonic map in the following sense. Let $(M, g)$ and $(N, h)$ be two Riemannian manifolds and $\psi: M \rightarrow N$, a smooth map. The exponentially harmonic map is a critical point of the following functional

$$
E(\phi)=\int_{M} \exp (e(\psi)) d \mu(\psi),
$$

where $d \mu(\psi)$ is the volume element and $e(\psi)=\frac{1}{2} \frac{\partial \psi^{i}}{\partial x^{k}} \frac{\partial \psi^{j}}{\partial x^{l}} g^{k l} h_{i j}$ is the energy density of the map $\psi$. The exponentially harmonic map can be generalized to the exponential wave map

$$
\phi: \mathbb{R}^{n+1} \rightarrow(N, h)
$$

Received March 6, 2012.

2010 Mathematics Subject Classification. 35L60, 35L70.

Key words and phrases. quasilinear wave, weakly linearly degenerate, double null form.

This research was supported by the Chung-Ang University Research Grants in 2010. 
where $\mathbb{R}^{n+1}$ is Minkowski space equipped with the metric $-d t^{2}+d x_{1}^{2}+\cdots+d x_{n}^{2}$. Then exponential wave map is the critical point of the functional

$$
E(\phi)=\int_{\mathbb{R}^{n+1}} \exp (e(\phi)) d x d t
$$

where $e(\phi)=\frac{1}{2}\left(\frac{\partial \phi^{i}}{\partial x_{k}} \frac{\partial \phi^{j}}{\partial x_{k}} h_{i j}-\frac{\partial \phi^{i}}{\partial t} \frac{\partial \phi^{j}}{\partial t} h_{i j}\right)$ is the energy density of the map $\phi$.

Some exact solutions of exponential wave maps were investigated in [8] and geometric aspects discussed in [2]. They also described the applications of exponential wave maps in Kaluza-Klein gravity [15] by coupling gravitational fields with exponential scalar fields.

We study the initial value problem of the exponential wave equation (1.1) which is Euler-Lagrange equation of (1.2) with $N=\mathbb{R}$. In particular, we are interested in the global solution for small initial data. According to the dimension $n$, we apply a different method to prove the existence of global solution.

The equation (1.1) in $\mathbb{R}^{1+1}$ reads as

$$
\left(1-\phi_{t}^{2}\right) \phi_{t t}+2 \phi_{t} \phi_{x} \phi_{t x}-\left(1+\phi_{x}^{2}\right) \phi_{x x}=0 .
$$

Denoting $\omega=\phi_{x}$ and $v=\phi_{t}$, the Cauchy problem of equation (1.3) can be formulated in the form

$$
\partial_{t}\left(\begin{array}{l}
\omega \\
v
\end{array}\right)+\left(\begin{array}{cc}
0 & -1 \\
\frac{\omega^{2}+1}{v^{2}-1} & \frac{2 v \omega}{1-v^{2}}
\end{array}\right) \partial_{x}\left(\begin{array}{c}
\omega \\
v
\end{array}\right)=0,
$$

with initial data $\omega(0, x)=\omega_{0}(x)$ and $v(0, x)=v_{0}(x)$. Our first result is as follows.

Theorem 1.1. For the initial data $\omega_{0}, v_{0} \in C^{1}(\mathbb{R})$ satisfying

$$
\theta \equiv \sup _{x \in \mathbb{R}}\left\{(1+|x|)^{1+\mu}\left(\left|\omega_{0}(x)\right|+\left|v_{0}(x)\right|+\left|\omega_{0}^{\prime}(x)\right|+\left|v_{0}^{\prime}(x)\right|\right)\right\}<\infty,
$$

there exists $\theta_{0}>0$ so small that if $\theta \in\left[0, \theta_{0}\right]$, then the equation (1.4) has global solution.

To prove Theorem 1.1 we observe that the first order quasilinear hyperbolic system (1.4) has so-called 'weakly linearly degenerate' structure. Furthermore we show that the initial value problem of (1.3) around non-trivial solution $\phi(t, x)=a x+b(a \neq 0)$ develops the finite time blow-up.

For the Cauchy problem of the equation (1.1) in $\mathbb{R}^{n+1}(n \geq 2)$, we have the following result.

Theorem 1.2. Let $n \geq 2$ and $f, g \in C_{0}^{\infty}\left(\mathbb{R}^{n}\right)$ be given. We consider the initial value problem of the equation (1.1) in $\mathbb{R}^{n+1}$ with the initial data

$$
\phi(0, x)=\varepsilon f(x), \quad \partial_{t} \phi(0, x)=\varepsilon g(x) .
$$

Then, there exists sufficiently small $\varepsilon_{0}$ such that if $\varepsilon \leq \varepsilon_{0}$, then the smooth solution of the equation (1.1) exists globally. 
The proof actually does not require compact support of the initial data; appropriate decays at infinity is enough. Furthermore the asymptotic behavior of the solution can be obtained in the middle of proof. To prove Theorem 1.2 we make use of a vector field method [9]. Especially we follow the idea of $\mathrm{H}$. Lindblad [13] in which the global existence of the minimal surface equation is proved for small initial data.

We raise and lower indices with the Minkowski metric $\eta=\left(\eta_{\alpha \beta}\right)=\operatorname{diag}(-1$, $1, \ldots, 1)$. We also use the summation convention for the repeated indices. Greek indices are used to denote $0, \ldots, n$, while Latin indices are for $1, \ldots, n$.

In Section 2, we collect some preliminaries. Theorem 1.1 is proved in Section 3 and Theorem 1.2 in Section 4.

\section{Preliminaries}

For the completeness of exposition, we briefly describe the known facts without proofs. The proofs can be found in references quoted below.

For the first order quasilinear hyperbolic system, we refer to $[1,10]$. Consider Cauchy problem

$$
u_{t}+A(u) u_{x}=0, u(0, x)=\varphi(x),
$$

where $A(u)=\left(a_{i j}(u)\right)$ is an $m \times m$ matrix with suitably smooth elements $a_{i j}(u)$, and $\varphi(x)$ is a $C^{1}$ vector function of $x$. Suppose that $A(u)$ has $m$ real eigenvalues $\lambda_{1}(u), \ldots, \lambda_{m}(u)$ and a complete system of right eigenvectors $A(u) r_{i}(u)=\lambda_{i}(u) r_{i}(u)$. We also suppose that the system (2.1) is strictly hyperbolic in a neighborhood of $u=0$, namely

$$
\lambda_{1}(0)<\cdots<\lambda_{m}(0) .
$$

Definition 2.1. The $i$-th characteristic $\lambda_{i}(u)$ is weakly linearly degenerate, if, along the $i$-th characteristic trajectory $u=u^{(i)}(s)$ passing through $u=0$, defined by

$$
\frac{d u}{d s}=r_{i}(u), \quad s=0: u=0
$$

we have

$$
\nabla \lambda_{i}(u) r_{i}(u) \equiv 0 \quad \text { for small }|u|
$$

namely,

$$
\lambda_{i}\left(u^{(i)}(s)\right) \equiv \lambda_{i}(0) \quad \text { for small }|s|
$$

If all characteristics are weakly linearly degenerate, then system (2.1) is called to be weakly linearly degenerate.

The following theorem [11] will be made use of.

Theorem 2.2. Suppose that (2.2) holds and system (2.1) is weakly linearly degenerate. Suppose furthermore that $A(u) \in C^{2}$ in a neighborhood of $u=0$ 
and $\varphi$ is a $C^{1}$ vector function satisfying that there exists a constant $\mu>0$ such that

$$
\theta \equiv \sup _{x \in \mathbb{R}}\left\{(1+|x|)^{1+\mu}\left(|\varphi(x)|+\left|\varphi^{\prime}(x)\right|\right)\right\}<\infty .
$$

Then there exists $\theta_{0}>0$ so small that for any given $\theta \in\left[0, \theta_{0}\right]$, Cauchy problem (2.1) admits a unique global $C^{1}$ solution $u=u(t, x)$ on $t \geq 0$.

The next review is for the proof of Theorem 1.2. We refer to $[9,16]$. Let us introduce the vector fields

$$
\Omega_{\mu \nu}=x_{\mu} \partial_{\nu}-x_{\nu} \partial_{\mu}, \quad S=x^{\mu} \partial_{\mu}
$$

which are the rotation and the scaling operators in $\mathbb{R}^{n+1}$. In what follows, the vector fields $\partial_{\mu}, S, \Omega_{\mu \nu}(\mu<\nu)$ are denoted by $\Gamma_{k}, k=0, \ldots, \frac{(n+1)(n+2)}{2}$. We use the multi-index notation $\Gamma^{I}=\Gamma_{1}^{I_{1}} \cdots \Gamma_{m}^{I_{m}}$. We recall the commutation relations between wave operator $\square=\partial_{\mu} \partial^{\mu}$ and $\Gamma$.

$$
\begin{aligned}
{[\square, S] } & =2 \square, \\
{\left[\square, \partial_{\mu}\right] } & =0,0 \leq \mu \leq n, \\
{\left[\square, \Omega_{\mu \nu}\right] } & =0,0 \leq \mu<\nu \leq n .
\end{aligned}
$$

The following relations can be found

$$
\left[\Gamma_{k}, \partial_{\nu}\right]=a_{\nu k}^{\mu} \partial_{\mu}
$$

for some suitable constant coefficients $a_{\nu k}^{\mu}$. Let us introduce the null forms

$$
\begin{gathered}
\tilde{Q}(\phi, \psi)=\partial_{t} \phi \partial_{t} \psi-\sum_{i=1}^{n} \partial_{i} \phi \partial_{i} \psi \\
Q_{\alpha \beta}(\phi, \psi)=\partial_{\alpha} \phi \partial_{\beta} \psi-\partial_{\alpha} \psi \partial_{\beta} \phi \quad \text { for } \alpha, \beta=0,1, \ldots, n .
\end{gathered}
$$

Let $Q$ stand for any of the above null forms. Then the following commutation relations hold

$$
\Gamma Q(\phi, \psi)=Q(\Gamma \phi, \psi)+Q(\phi, \Gamma \psi)+b^{\mu \nu} Q_{\mu \nu}(\phi, \psi)
$$

for some constants $b^{\mu \nu}$. The following lemma in [16] will be used to give time decay factor $\frac{1}{1+t}$ to the quadratic terms $Q$.

Lemma 2.3. Let $Q$ be one of the null forms in (2.3). Then if $t>0$,

$$
|Q(\phi, \psi)(t, x)| \leq \frac{C}{1+t+|x|} \sum_{|I|=1}\left|\Gamma^{I} \phi(t, x)\right| \sum_{|I|=1}\left|\Gamma^{I} \psi(t, x)\right| .
$$

Combining the above lemma with (2.4), one can easily verify.

Corollary 2.4. Let $Q$ be one of the null forms in (2.3). Then if $t>0$,

$$
(1+t+|x|) \sum_{|I| \leq M}\left|\Gamma^{I} Q(\phi, \psi)\right| \leq C_{M} \sum_{1 \leq|I| \leq M+1}\left|\Gamma^{I} \phi\right| \sum_{1 \leq|I| \leq \frac{M+2}{2}}\left|\Gamma^{I} \psi\right|
$$




$$
+C_{M} \sum_{1 \leq|I| \leq M+1}\left|\Gamma^{I} \psi\right| \sum_{1 \leq|I| \leq \frac{M+2}{2}}\left|\Gamma^{I} \phi\right| .
$$

To estimate $L^{2}$ norm of the solution itself $\phi$, use will be made of the following lemma, the proof of which is in $[12,13]$.

Lemma 2.5. If u solve the Cauchy problem

$$
\square u=\sum_{\alpha=0}^{n} \partial_{\alpha} G_{\alpha}, u(0,)=\varepsilon f, u_{t}(0,)=\varepsilon g,
$$

then

$$
\|u(t, \cdot)\|_{L^{2}} \leq \sum_{\alpha=0}^{n} \int\left\|G_{\alpha}(s, \cdot)\right\|_{L^{2}} d s+C\left(f, g, G_{0}(0, \cdot)\right) m(t) \varepsilon .
$$

where $m(t)=1$ if $n \geq 3, m(t)=\log (2+t)$ if $n=2$ and $C\left(f, g, G_{0}(0, \cdot)\right)$ is some constant depending on some weighted Sobolev norm of initial data $f$ and $g$.

We also use the following $L^{1}-L^{\infty}$ estimate in [6].

Lemma 2.6. The solution $u$ of

$$
\square u(t, x)=F(t, x), \quad u(0, \cdot)=\varepsilon f, \quad \partial_{t} u(0, \cdot)=\varepsilon g, \quad(t, x) \in \mathbb{R}^{n+1}
$$

satisfies

$|u(t, x)| \leq C(1+t+|x|)^{-\frac{n-1}{2}}\left(\sum_{|I| \leq n-1} \int_{0}^{t}\left\|\frac{\Gamma^{I} F(s, \cdot)}{(1+s+|\cdot|)^{\frac{n-1}{2}}}\right\|_{L^{1}} d s+C(f, g) \varepsilon\right)$.

\section{Proof of Theorem 1.1}

In this section we are interested in the exponential wave equation in $\mathbb{R}^{1+1}$.

$$
\left(1-\phi_{t}^{2}\right) \phi_{t t}+2 \phi_{t} \phi_{x} \phi_{t x}-\left(1+\phi_{x}^{2}\right) \phi_{x x}=0 .
$$

Applying the formulation of the first order hyperbolic system, we show the global existence of the solution to (3.1) for sufficiently decaying initial data. Let $\omega=\phi_{x}, v=\phi_{t}$ and $U=(\omega, v)^{T}$. Then the equation (3.1) can be written in the form

$$
\partial_{t} U+A(U) \partial_{x} U=0, \quad \text { with } \quad A(U)=\left(\begin{array}{cc}
0 & -1 \\
\frac{\omega^{2}+1}{v^{2}-1} & \frac{2 v \omega}{1-v^{2}}
\end{array}\right) .
$$

The following eigenvalues of $A(U)$ in (3.2) are well-defined distinct real values in the neighborhood of $(\omega, v)=(0,0)$

$$
\lambda_{1}=\frac{v \omega}{1-v^{2}}-\frac{\sqrt{1+\omega^{2}-v^{2}}}{1-v^{2}}, \quad \lambda_{2}=\frac{v \omega}{1-v^{2}}+\frac{\sqrt{1+\omega^{2}-v^{2}}}{1-v^{2}} .
$$

The corresponding right eigenvectors $A r_{i}=\lambda_{i} r_{i}$ are

$$
r_{1}=\left(v^{2}-1, v \omega-\sqrt{1+\omega^{2}-v^{2}}\right)^{T}, \quad r_{2}=\left(v^{2}-1, v \omega+\sqrt{1+\omega^{2}-v^{2}}\right)^{T} .
$$


A calculation shows that

$$
\begin{aligned}
& \nabla \lambda_{1} \cdot r_{1} \\
= & \left(\partial_{\omega} \lambda_{1}, \partial_{v} \lambda_{1}\right) \cdot r_{1} \\
= & \left(\frac{v}{1-v^{2}}-\frac{\omega}{\left(1-v^{2}\right) \sqrt{1+\omega^{2}-v^{2}}}\right) \cdot\left(v^{2}-1\right) \\
& +\left(\frac{\omega\left(v^{2}+1\right)}{\left(1-v^{2}\right)^{2}}+\frac{v\left(1-v^{2}\right)-2 v\left(1+\omega^{2}-v^{2}\right)}{\left(1-v^{2}\right)^{2} \sqrt{1+\omega^{2}-v^{2}}}\right) \cdot\left(v \omega-\sqrt{1+\omega^{2}-v^{2}}\right) .
\end{aligned}
$$

The above quantity is not identically zero near $(\omega, v)=(0,0)$, i.e., not linearly degenerate in the sense of P. D. Lax. Here the 'weakly linearly degenerate' comes in the story. We can solve the following system of ordinary differential equations on small $|s|$

$$
\frac{d}{d s}\left(\begin{array}{l}
\omega \\
v
\end{array}\right)=\left(\begin{array}{c}
v^{2}-1 \\
v \omega-\sqrt{1+\omega^{2}-v^{2}}
\end{array}\right) \text { with }\left(\begin{array}{l}
\omega(0) \\
v(0)
\end{array}\right)=\left(\begin{array}{l}
0 \\
0
\end{array}\right) .
$$

To show that (3.2) is weakly linearly degenerate we have to check $\frac{d}{d s}\left(\nabla \lambda_{1}\right.$. $\left.r_{1}\right)(s)=0$ for small $|s|$. Then $\nabla \lambda_{1} \cdot r_{1}(s)=\nabla \lambda_{1} \cdot r_{1}(0)=0$. In fact, let $f$ be the solution to the ordinary differential equation

$$
\frac{d}{d s} f(s)=f^{2}(s)-1, f(0)=0 .
$$

We can check that $v(s)=f(s)$ and $\omega(s)=f(s)$ are the solution to (3.3) by the uniqueness of local solution. Then we have $\frac{d}{d s}\left(\nabla \lambda_{1} \cdot r_{1}\right)(s)=0$ along the first characteristic trajectory. The corresponding quantity for the eigenvalue $\lambda_{2}$

$$
\begin{aligned}
& \nabla \lambda_{2} \cdot r_{2} \\
= & \left(\frac{v}{1-v^{2}}+\frac{\omega}{\left(1-v^{2}\right) \sqrt{1+\omega^{2}-v^{2}}}\right) \cdot\left(v^{2}-1\right) \\
& +\left(\frac{\omega\left(v^{2}+1\right)}{\left(1-v^{2}\right)^{2}}-\frac{v\left(1-v^{2}\right)-2 v\left(1+\omega^{2}-v^{2}\right)}{\left(1-v^{2}\right)^{2} \sqrt{1+\omega^{2}-v^{2}}}\right) \cdot\left(v \omega+\sqrt{1+\omega^{2}-v^{2}}\right)
\end{aligned}
$$

vanishes along second characteristic trajectory

$$
\frac{d}{d s}\left(\begin{array}{l}
\omega \\
v
\end{array}\right)=\left(\begin{array}{c}
v^{2}-1 \\
v \omega+\sqrt{1+\omega^{2}-v^{2}}
\end{array}\right) \text { with }\left(\begin{array}{c}
\omega(0) \\
v(0)
\end{array}\right)=\left(\begin{array}{l}
0 \\
0
\end{array}\right)
$$

by putting $\omega(s)=-g(s)$ and $v(s)=g(s)$, where $g$ is defined by $\frac{d}{d s} g(s)=$ $1-g^{2}(s), g(0)=0$. Therefore we can check that system (3.2) is weakly linearly degenerate. Applying Theorem 2.2 we can prove Theorem 1.1.

We next consider the Cauchy problem around non-trivial static solutions of (3.1). The finite time blow up result will be obtained. The assumption $\phi_{t} \equiv 0$ implies that $\phi_{x x}=0$ which leads to a static solution $a x+b$ for some constants $a$ and $b$. Substitute $\phi(x, t)=a x+b+h(x, t)$ into (3.1) then we obtain

$$
\left(1-h_{t}^{2}\right) h_{t t}+2\left(a+h_{x}\right) h_{t} h_{t x}-\left(1+\left(a+h_{x}\right)^{2}\right) h_{x x}=0 .
$$


Let $\omega=h_{x}, v=h_{t}$ and $U=(\omega, v)^{T}$. Then we have

$$
\partial_{t} U+A_{a}(U) \partial_{x} U=0, \quad \text { with } \quad A_{a}(U)=\left(\begin{array}{cc}
0 & -1 \\
\frac{(a+\omega)^{2}+1}{v^{2}-1} & \frac{2 v(a+\omega)}{1-v^{2}}
\end{array}\right) .
$$

Let $\lambda_{j}^{a}, r_{j}^{a}$ be the corresponding eigenvalue, eigenvector of $A_{a}$. The same calculation as before shows $\nabla \lambda_{1}^{a} \cdot r_{1}^{a} \neq 0$ in the neighborhood of $(\omega, v)=(0,0)$. In fact we can check that $\nabla \lambda_{1}^{a} \cdot r_{1}^{a}(0,0)=\frac{a}{\sqrt{1+a^{2}}}-a \sqrt{1+a^{2}} \neq 0$ for $a \neq 0$. Therefore the well-known results of F. John [7] say that the first order derivatives of the $C^{2}$ solution to the Cauchy problem (3.4) with $a \neq 0$ must blow up in a finite time.

\section{Proof of Theorem 1.2}

In this section we prove Theorem 1.2 which implies the stability of trivial static solution of the equation (1.1). In fact, static assumption $\partial_{t} \phi \equiv 0$ leads to elliptic equation

$$
\Delta \phi+\sum_{i, j=1}^{n} \partial_{i} \phi \partial_{j} \phi \partial_{i j} \phi=0
$$

which was derived from the variational principle of $\int_{\mathbb{R}^{n}} \exp \left(\frac{1}{2}|\nabla u|^{2}\right) d x$ in [5]. A real function is called an exponentially harmonic function if it satisfies (4.1). It was proved in [14] that any bounded exponentially harmonic function must be constant. To show the stability of trivial solution $\phi \equiv 0$, we consider the following initial value problem.

$$
\begin{gathered}
\frac{\partial}{\partial t}\left(\partial_{t} \phi \exp \left(\frac{1}{2}|\nabla \phi|^{2}-\frac{1}{2}\left(\partial_{t} \phi\right)^{2}\right)\right)-\sum_{i=1}^{n} \frac{\partial}{\partial x_{i}}\left(\partial_{i} \phi \exp \left(\frac{1}{2}|\nabla \phi|^{2}-\frac{1}{2}\left(\partial_{t} \phi\right)^{2}\right)\right)=0, \\
\phi(0, x)=\varepsilon f(x), \quad \partial \phi(0, x)=\varepsilon g(x),
\end{gathered}
$$

where $n \geq 2$ and $f, g \in C_{0}^{\infty}\left(\mathbb{R}^{n}\right)$.

First of all we turn into the question of local existence of solution. We can rewrite the equations (4.2) in the following form

$$
\square \phi+\mathcal{C}^{\alpha \beta} \partial_{\alpha \beta} \phi=0,
$$

where $\mathcal{C}^{\alpha \beta}=\mathcal{C}^{\alpha \beta}(\partial \phi)$ are polynomials of order 2 . We also note that $\mathcal{C}(0)=0$ and

$$
\mathcal{C}^{\alpha \beta}(\partial \phi)=\mathcal{C}^{\beta \alpha}(\partial \phi)
$$

To prove the local well-posedness in the suitable Sobolev spaces, let us use the energy estimate for the following equation

$$
\square \phi+\mathcal{C}^{\alpha \beta}(t, x) \partial_{\alpha \beta} \phi=f,
$$


under the assumptions $|\mathcal{C}|=\sum\left|\mathcal{C}^{\alpha \beta}\right| \leq \frac{1}{2}$ and $\mathcal{C}^{\alpha \beta}(t, x)=\mathcal{C}^{\beta \alpha}(t, x)$. Multiplying $\partial_{t} \phi$ and integrating over $\mathbb{R}^{n}$, we obtain

$$
\int_{\mathbb{R}^{n}} \partial_{t} \phi \square \phi+\partial_{t} \phi \mathcal{C}^{\alpha \beta} \partial_{\alpha \beta} \phi=\int_{\mathbb{R}^{n}} \partial_{t} \phi f .
$$

Using the fact that $\mathcal{C}^{\alpha \beta}=\mathcal{C}^{\beta \alpha}$ and integrating by parts, we deduce

$$
\begin{gathered}
\partial_{t} \int_{\mathbb{R}^{n}} \frac{1}{2}|\partial \phi|^{2}+\mathcal{C}^{i j} \partial_{i} \phi \partial_{j} \phi-\mathcal{C}^{00} \partial_{0} \phi \partial_{0} \phi \\
=\int_{\mathbb{R}^{n}} \partial_{0} \mathcal{C}^{i j} \partial_{i} \phi \partial_{j} \phi-\partial_{i} \mathcal{C}^{i j} \partial_{0} \phi \partial_{j} \phi-\partial_{j} \mathcal{C}^{i j} \partial_{0} \phi \partial_{i} \phi \\
\quad-\int_{\mathbb{R}^{n}} \partial_{0} \phi f+\partial_{i} \mathcal{C}^{00} \partial_{0} \phi \partial_{0} \phi+\partial_{0} \mathcal{C}^{00} \partial_{0} \phi \partial_{0} \phi .
\end{gathered}
$$

Integrating over $[0, T]$, taking into account the smallness of $\mathcal{C}^{\alpha \beta}$ and applying Hölder inequality on the right hand side of (4.6), we obtain

$$
\begin{aligned}
\|\partial \phi(t)\|_{L^{2}} \leq & C \exp \left(\int_{0}^{t}|\partial \mathcal{C}(\tau)| d \tau\right)\|\partial \phi(0)\|_{L^{2}} \\
& +C \int_{0}^{t} \exp \left(\int_{s}^{t}|\partial \mathcal{C}(\tau)| d \tau\right)\|f(s, \cdot)\|_{L^{2}} d s,
\end{aligned}
$$

where $|\partial \mathcal{C}(\tau)|=\sum \sup _{x}\left|\partial \mathcal{C}^{\alpha \beta}(\tau, x)\right|$ and the constant $C$ depends only on the $L^{\infty}$ norm of $\mathcal{C}^{\alpha \beta}$. Differentiation of (4.5) with respect to time or the spatial variable shows that each partial derivative of $\phi$ satisfies an equation with the same principal part as (4.5) and remainder terms depending on the derivatives of $\mathcal{C}, f$ and $\phi$ of lower order. Thus one can obtain energy estimates of for the higher order derivatives of $\phi$.

Now we will present the proof of Theorem 1.2. Below we concentrate on the case of $n=2$. The case $n \geq 3$ is similar and even simpler due to the faster decays near infinity of the solutions to the linear wave equation. We also mention that the main part of our proof is a direct adaptation of the argument in [13].

We can rewrite (4.2) as wave equation with a right hand side in divergence form

$$
\square \phi=\partial^{\mu}\left(\left(1-e^{-\frac{1}{2} \tilde{Q}(\phi, \phi)}\right) \partial_{\mu} \phi\right),
$$

where we denote $\exp \left(\frac{1}{2}|\nabla \phi|^{2}-\frac{1}{2}\left|\partial_{t} \phi\right|^{2}\right)=e^{-\frac{1}{2} \tilde{Q}(\phi, \phi)}$. Applying $\Gamma^{I}$ to the both sides of (4.8), we get

$$
\square \Gamma^{I} \phi=\sum_{\mu=0}^{2} \partial_{\mu}\left(\sum G_{i_{1}, \cdot, i_{k}, I_{1}, \cdot, I_{k}}(Q) \partial_{i_{1}} \Gamma^{I_{1}} \phi \cdots \partial_{i_{k}} \Gamma^{I_{k}} \phi\right) .
$$

Note that a right hand side of (4.9) is in the divergence form and $G_{i_{1}, \cdot, i_{k}, I_{1}, \cdot, I_{k}}(Q)$ is bounded function for small $Q$. 
Also we can alternatively write (4.2) as a wave equation with the double null forms

$$
\square \phi+\frac{1}{2} \tilde{Q}(\phi, \tilde{Q}(\phi, \phi))=0
$$

Applying $\Gamma^{I}$, we obtain

$$
\begin{aligned}
& \square \Gamma^{I} \phi-\frac{1}{2} \tilde{Q}\left(\phi, \tilde{Q}\left(\phi, \Gamma^{I} \phi\right)\right) \\
= & \sum_{\substack{k \geq 3,\left|I_{1}\right|+\cdots+\left|I_{k}\right| \leq|I|+1 \\
\left|I_{i}\right| \leq \frac{|I|+1}{2}, i<k,\left|I_{k}\right| \leq|I|}} H_{i_{1}, \ldots, i_{k}, I_{1}, \ldots, I_{k}}(\phi, Q(\phi, \phi))\left(\partial_{i_{1}} \Gamma^{I_{1}} \phi\right) \cdots\left(\partial_{i_{1}} \Gamma^{I_{1}} \phi\right),
\end{aligned}
$$

where $H$ is a bounded function for small $\phi, Q(\phi, \phi)$ and the coefficients of the highest order terms are symmetric in the sense of (4.4). Finally we write (4.2) in the form suitable for applying the $L^{1}-L^{\infty}$ estimate of Lemma 2.6.

$$
\begin{aligned}
\square \Gamma^{I} \phi=\sum_{\substack{k \geq 3 \\
\left|I_{1}\right|+\cdots+\left|I_{k}\right| \leq|I|}} & K_{I_{1}, \cdots, I_{k}}^{i_{0}, \cdots, i_{k}}(Q(\phi, \phi)) Q^{i_{0} i_{1}}\left(\Gamma^{I_{1}} \phi, Q^{i_{0} i_{1}}\left(\Gamma^{I_{2}} \phi, \Gamma^{I_{3}} \phi\right)\right) \\
& \times Q^{i_{4} i_{5}}\left(\Gamma^{I_{4}} \phi, \Gamma^{I_{5}} \phi\right) \cdots Q^{i_{k-1} i_{k}}\left(\Gamma^{I_{k-1}} \phi, \Gamma^{I_{k}} \phi\right),
\end{aligned}
$$

where $i_{j-1} i_{j}$ is the index for denoting the several terms under a fixed $I_{1}, \ldots, I_{k}$ and $K$ is a bounded function for small $Q(\phi, \phi)$.

We will prove that following bounds are guaranteed all the time if $L$ is sufficiently large and $\varepsilon$ is sufficiently small.

$$
\begin{aligned}
& M_{1}(t)=\sum_{|I| \leq N}\left\|\partial \Gamma^{I} \phi(t, \cdot)\right\|_{L^{2}} \leq L \varepsilon(1+t)^{\delta} \\
& M_{2}(t)=\sum_{|I| \leq N}\left\|\Gamma^{I} \phi(t, \cdot)\right\|_{L^{2}} \leq L \varepsilon(1+t)^{\delta} \\
& N_{1}(t)=\sum_{|J| \leq \frac{N+1}{2}+1}\left\|\Gamma^{J} \phi(t, \cdot)\right\|_{L^{\infty}} \leq L \varepsilon(1+t)^{-\frac{1}{2}},
\end{aligned}
$$

where $0<\delta<\frac{1}{2}$ fixed. Applying the energy estimates (4.7) to (4.11), we have (4.14)

$$
M_{1}(t) \leq C \varepsilon \exp \left(\int_{0}^{t} N_{1}(\tau)^{2} d \tau\right)+\int_{0}^{t} \exp \left(\int_{s}^{t} N_{1}(\tau)^{2} d \tau\right) C\left(N_{1}(s)\right) N_{1}(s)^{2} M_{1}(s) d s .
$$

Lemma 2.5 applied to $(4.9)$ gives

$$
M_{2}(t) \leq C\left(C(f, g) m(t) \varepsilon+\int_{0}^{t} C\left(N_{1}(s)\right) N_{1}(s)^{2} M_{1}(s) d s\right) .
$$


Finally, Lemma 2.6 applied to (4.12), Lemma 2.3 and Cauchy Schwartz inequality give

$$
N_{1}(t) \leq C(1+t)^{-\frac{1}{2}}\left(C(f, g) \varepsilon+\int_{0}^{t} \frac{N_{1}(s)}{(1+s)^{\frac{1}{2}+1}}\left(M_{1}(s)+M_{2}(s)\right)^{2} d s\right)
$$

if $\frac{N+1}{2}+1+n-1 \leq N$, i.e., $N \geq 2 n+1$. We note that the null condition produced an extra power of $(1+s)^{-1}$ in the integral (4.16). From the bound (4.13) we can get

$$
\exp \left(\int_{s}^{t} N_{1}(\tau)^{2} d \tau\right) \leq \exp \left(\varepsilon^{2} L^{2} \int_{s}^{t}(1+\tau)^{-1} d \tau\right)=\left(\frac{1+t}{1+s}\right)^{\varepsilon^{2} L^{2}},
$$

so it follows from $(4.14)$

$$
M_{1}(t) \leq C \varepsilon(1+t)^{K^{2} \varepsilon^{2}}+\int_{0}^{t} C \varepsilon^{3} L^{3}\left(\frac{1+t}{1+s}\right)^{\varepsilon^{2} L^{2}}(1+s)^{\delta-1} d s \leq \frac{L \varepsilon}{2}(1+t)^{\delta},
$$

if $L$ is sufficiently large and $\varepsilon$ is sufficiently small. Also, from (4.15) we get

$$
M_{2}(t) \leq C \varepsilon \log (2+t)+\int_{0}^{t} C \varepsilon^{3} L^{3}(1+s)^{\delta-1} d s \leq \frac{L \varepsilon}{2}(1+t)^{\delta},
$$

if $L$ is sufficiently large and $\varepsilon$ is sufficiently small. Finally from (4.16) we get

$$
N_{1}(t) \leq C \varepsilon(1+t)^{-\frac{1}{2}}+(1+t)^{-\frac{1}{2}} \int_{0}^{t} C \varepsilon^{3} L^{3}(1+s)^{2 \delta-2} d s \leq \frac{L \varepsilon}{2}(1+t)^{-\frac{1}{2}},
$$

if $L$ is sufficiently large and $\varepsilon$ is sufficiently small, since $0<\delta<\frac{1}{2}$. Consequently, we get a bound of $N_{1}(t)$ smaller than the assumption. Therefore the solution can be extended by continuity argument. If we assume the maximal time of existence $T=T_{*}(<\infty)$, the above result contradicts the maximality of $T_{*}$. We obtain the global existence of exponential wave equation for small initial data.

\section{References}

[1] S. Alinhac, Hyperbolic Partial Differential Equations, Universitext, Springer, Dordrecht, 2009.

[2] Y.-J. Chiang and Y.-H. Yang, Exponential wave maps, J. Geom. Phys. 57, (2007) no. $12,2521-2532$.

[3] J. Eells and L. Lemaire, Another report on harmonic maps, Bull. London Math. Soc. 20 (1988), no. 5, 385-524

[4] - Some properties of exponentially harmonic maps, Partial differential equations, Part 1, 2 (Warsaw, 1990), 129-136, Banach Center Publ., 27, Part 1, 2, Polish Acad. Sci., Warsaw, 1992.

[5] D. Gilbarg and N. S. Trudinger, Elliptic Partial Differential Equations of Second Order, Springer Verlag, 1977.

[6] L. Hörmander, Lectures on Nonlinear Hyperbolic Differential Equations, Springer Verlag, 1997.

[7] F. John, Formation of singularities in one-dimensional nonlinear wave propagation, Comm. Pure Appl. Math. 27 (1974), 377-405. 
[8] A. D. Kanfon, A. Fuzfa, and D. Lambert, Some examples of exponentially harmonic maps, J. Phys. A 35 (2002), no. 35, 7629-7639.

[9] S. Klainerman, The null condition and global existence to nonlinear wave equations, Nonlinear systems of partial differential equations in applied mathematics, Part 1 (Santa Fe, N.M., 1984), 293-326, Lectures in Appl. Math., 23, Amer. Math. Soc., Providence, RI, 1986.

[10] D. Kong, Cauchy Problem for Quasilinear Hyperbolic Systems, MSJ Memoirs, 6. Mathematical Society of Japan, Tokyo, 2000.

[11] T.-T. Li, Y. Zhou, and D. Kong, Global classical solutions for general quasilinear hyperbolic systems with decay initial data, Nonlinear Anal. 28 (1997), no. 8, 1299-1332.

[12] H. Lindblad, On the lifespan of solutions of nonlinear wave equations with small initial data, Comm. Pure Appl. Math. 43 (1990), no. 4, 445-472.

[13] H. Lindblad, A remark on global existence for small initial data of the minimal surface equation in Minkowskian space time, Proc. Amer. Math. Soc. 132 (2004), no. 4, 10951102.

[14] M. C. Hong, Liouville theorems for exponentially harmonic functions on Riemannian manifolds, Manuscripta Math. 77 (1992), no. 1, 41-46.

[15] J. M. Overduin and P. S. Wesson, Kaluza-Klein gravity, Phys. Rep. 283 (1997), no. 5-6, 303-378.

[16] C. D. Sogge, Lectures on Nonlinear Wave Equations, International Press Incorporated, Boston, 1995.

Department of Mathematics

Chung-Ang University

SeOul 156-756, Korea

E-mail address: huh@cau.ac.kr 